\title{
Selection of a malignant subpopulation from a colorectal cancer cell line
}

\author{
PEI-LUN LAI ${ }^{1,2^{*}}$, TING-CHUN CHEN ${ }^{1 *}$, CHUN-YEN FENG $^{1 *}$, HSUAN LIN $^{2}$, CHI-HOU NG $^{2,3}$, \\ YUN CHEN $^{1}$, MICHAEL HSIAO ${ }^{2}$, JEAN LU ${ }^{2-6}$ and HSIAO-CHUN HUANG ${ }^{1,3,7,8}$ \\ ${ }^{1}$ Institute of Molecular and Cellular Biology, College of Life Science, \\ National Taiwan University, Taipei 10617; ${ }^{2}$ Genomics Research Center, Academia Sinica, Taipei 11529; \\ ${ }^{3}$ Genome and Systems Biology Degree Program, College of Life Science, National Taiwan University, Taipei 10617; \\ ${ }^{4}$ National RNAi Platform/National Core Facility Program for Biotechnology, Taipei 11529; ${ }^{5}$ Department of Life Science, \\ Tzu Chi University, Hualien 970; ${ }^{6}$ Graduate Institute of Medical Sciences, National Defense Medical Center, Taipei 11490; \\ ${ }^{7}$ Department of Life Science, College of Life Science, National Taiwan University, Taipei 10617; ${ }^{8}$ Graduate \\ Institute of Electronics Engineering, College of Electrical Engineering and Computer Science, \\ National Taiwan University, Taipei 10617, Taiwan, R.O.C.
}

Received November 21, 2019; Accepted June 16, 2020

DOI: $10.3892 / \mathrm{ol} .2020 .11829$

\begin{abstract}
Colorectal cancer (CRC) is a leading cause of cancer-associated mortality worldwide; therefore, there is an emerging need for novel experimental models that allow for the identification and validation of biomarkers for CRC-specific progression. In the present study, a repeated sphere-forming assay was used as a strategy to select a malignant subpopulation from a CRC cell line, namely HCT116. The assay was validated by confirming that canonical stemness markers were upregulated in the sphere state at every generation of the selection assay. The resulting subpopulation, after eight rounds of selection, exhibited increased sphere-forming capacity in vitro and increased tumorigenicity in vivo. Furthermore, dipeptidase 1 (DPEP1) was identified as the major differentially expressed gene in the selected clone, and its depletion suppressed the elevated sphere-forming capacity in vitro and tumorigenicity
\end{abstract}

Correspondence to: Dr Jean Lu, Genomics Research Center, Academia Sinica, 128 Academia Road, Section 2, Nankang, Taipei 11529, Taiwan, R.O.C.

E-mail: jeanlu@gate.sinica.edu.tw

Dr Hsiao-Chun Huang, Institute of Molecular and Cellular Biology, College of Life Science, National Taiwan University, 1 Roosevelt Road, Section 4, Da'an, Taipei 10617, Taiwan, R.O.C.

E-mail: hsiaochun@ntu.edu.tw

*Contributed equally

Abbreviations: CRC, colorectal cancer; CSCs, cancer stem cells; DPEP1, dipeptidase 1; SDACs, sphere-derived adherent cells; SDCSCs, sphere-derived cancer stem cell-like cells

Key words: cell line, colorectal cancer, DPEP1, malignant subpopulation, sphere-forming in vivo. Overall, the present study established an experimental strategy to isolate a malignant subpopulation from a CRC cell line. Additionally, results from the present model revealed that DPEP1 may serve as a promising prognostic biomarker for CRC.

\section{Introduction}

Colorectal cancer (CRC) is the third most common cause of cancer-associated mortality worldwide and is a common neoplastic disease in the United State in 2020 (1,2). Similarly to numerous other types of cancer, the development of CRC from benign adenoma to malignant carcinoma is thought to result from the long-term accumulation of mutations during the course of disease progression (3). Since the survival rate of patients with CRC is closely associated with the time of diagnosis and the stage of the tumor (4), it is increasingly important to identify specific factors involved in disease progression that serve as prognostic markers. A good experimental model that covers a wide range of malignancies would facilitate the identification and validation of these CRC markers.

It has been proposed that cancer stem cells (CSCs), representing a subpopulation of cancer cells with self-renewal features that promote tumor growth and resistance to chemotherapy, are the most malignant subset of cells and are responsible for the recurrence and metastasis of CRC $(5,6)$. Sphere forming assay is a method that has been commonly used to identify CSCs (7-13). The history of the method can be traced to the late 1960s, when it was used to study neurogenesis in neural stem cells (7). Specifically, the sphere-forming assay was initially used to identify cells with higher neurogenic potential with respect to both clonality and multipotent differentiation (8). This assay has now been employed to investigate stem cells in a variety of normal tissues (8). Scientists have adopted the sphere-forming assay to form tumorspheres for numerous types of cancer, including prostate (9) brain (10), 
breast (11) and colorectal $(12,13)$ cancer. These tumorspheres have been reported to have similar self-renewal characteristics and to express the same canonical stemness markers, such as Nanog, as normal stem cells (14). A limitation, however, exists for the use of tumorspheres induced by sphere-forming assay as platforms to study CRC progression. Because these cancer cell lines are normally maintained in long-term adherent culture, the phenotypes induced by sphere-forming assays might only reflect properties of transient states (due to loss of attachment, difference in growth factor composition, etc), and not stable properties of the cells (15).

In order to overcome the aforementioned limitations, the present study used a repeated sphere-forming assay as a strategy to select a malignant subpopulation that was phenotypically stable. Conceptually, this strategy is similar to the previous establishment of a series of metastatic cell lines using repeated invasion assays $(16,17)$. Such model cell lines have been used to identify genes or cellular phenotypes that are associated with metastasis $(17,18)$. In the present study, as opposed to invasion assays, sphere-forming assays were used as repeated method for the generation of malignant lines. Through this repeated assay, a malignant clone from the CRC HCT116 cell line has been generated. RNA sequencing (RNA-seq) was used to compare transcriptomes between the selected clone and its parental cell line, and to identify any genes responsible for malignancy, both in vitro and in vivo.

\section{Materials and methods}

Cell culture, sphere-forming assay and preparation of sphere-derived adherent cells (SDACs). The human CRC HCT116 cell line was obtained from the Bioresource Collection and Research Center (Hsinchu, Taiwan) and maintained in McCoy's 5A medium (Gibco; Thermo Fisher Scientific, Inc.) supplemented with 10\% FBS (HyClone; GE Healthcare Life Sciences) and $1 \%$ penicillin/streptomycin at $37^{\circ} \mathrm{C}$ in a humidified incubator with $5 \% \mathrm{CO}_{2}$. For sphere formation, cells were plated at a density of 1,000 cells/well in 24-well ultra-low attachment plates (Corning, Inc.) and cultured in stem cell medium for 14 days at the aforementioned conditions. The stem cell medium consisted of serum-free DMEM/F12 medium (1:1; Gibco; Thermo Fisher Scientific, Inc.) supplemented with 50X B27 supplement (Gibco; Thermo Fisher Scientific, Inc.), 100X minimum essential medium non-essential amino acids (Gibco; Thermo Fisher Scientific, Inc.), 100 mM sodium pyruvate (Gibco; Thermo Fisher Scientific, Inc), 200 mM L-glutamine (Gibco; Thermo Fisher Scientific, Inc), $25 \mathrm{mg} / \mathrm{ml}$ insulin (Gibco; Thermo Fisher Scientific, Inc.), $20 \mathrm{ng} / \mathrm{ml}$ epidermal growth factor, $10 \mathrm{ng} / \mathrm{ml}$ basic fibroblast growth factor and $100 \mathrm{U} / \mathrm{ml}$ penicillin/streptomycin. To prepare SDACs, TrypLE Express (Gibco; Thermo Fisher Scientific, Inc.) was used to dissociate spheres by pipetting up and down repeatedly, and single cells were collected by centrifugation at $750 \mathrm{x}$ g for $5 \mathrm{~min}$ in room temperature. Subsequently, the cells were resuspended in DMEM/F12 medium supplemented with 10\% FBS and 1\% penicillin/streptomycin, and cultured for 14 days at $37^{\circ} \mathrm{C}$ in a humidified incubator with $5 \% \mathrm{CO}_{2}$ before the next sphere-forming assay round. For the time-course experiment to characterize the transient properties of SDCSCs, SDACs were prepared from G1S cells (using TrypLE Express to dissociate spheres and centrifugation at $750 \mathrm{x} g$ for $5 \mathrm{~min}$ to collect single cells) and resuspended in DMEM/F12 medium supplemented with $10 \%$ FBS and $1 \%$ penicillin/streptomycin, and cultured for 1, 3, 7 and 14 days before being harvested for total RNA and protein lysates for further analysis.

Microscopy. Phase-contrast images of sphere-derived CSC-like cells (SDCSCs) and SDACs were obtained using an inverted Axio Observer Z1 fluorescence microscope (Zeiss AG) (x10/0.30, x63/0.75) with a CoolSnap HQ2 camera (Photometrics; Taiwan Instrument Co., Ltd.). ZEN 2 (Zeiss AG) was used for image acquisition and subsequent analysis.

$R N A$ extraction and reverse transcription-quantitative $P C R$ (RT-qPCR). Total RNA was extracted from human CRC HCT116 cell line using phenol (Sigma-Aldrich; Merck $\mathrm{KGaA}$ )/chloroform (Avantor $\left.{ }^{\circledR}\right)$ and reverse-transcribed $\left(25^{\circ} \mathrm{C}\right.$ for $5 \mathrm{~min} ; 46^{\circ} \mathrm{C}$ for $20 \mathrm{~min}$; and $95^{\circ} \mathrm{C}$ for $1 \mathrm{~min}$ ) into cDNA using the iScript cDNA Synthesis kit (Bio-Rad Laboratories, Inc.). qPCR was performed using a SYBR Green kit (Roche Diagnostics) on a CFX96 qRT-PCR machine (Bio-Rad Laboratories, Inc.) as follows: $95^{\circ} \mathrm{C}$ for $10 \mathrm{sec} ; 57^{\circ} \mathrm{C}$ for $10 \mathrm{sec}$; $72^{\circ} \mathrm{C}$ for $30 \mathrm{sec} ; 40$ cycles. Relative mRNA levels were calculated according to the $2^{-\triangle \Delta \mathrm{CT}}$ method (19). GAPDH was used as the housekeeping gene. To validate the CSC-like properties of SDCSCs and the reliability of our assay, the canonical stemness marker Nanog was used, as it has been known to be upregulated in CRC CSCs (13). Another two stemness markers, octamer-binding transcription factor 4 (OCT4) and leucine-rich repeat-containing $\mathrm{G}$-protein coupled receptor 5 (LGR5), were used for further confirmation. The primer sequences were as follows: GAPDH forward, 5'-TGGTGA AGCAGGCGTCGGAG-3' and reverse, 5'-GGTGGGGGA CTGAGTGTGGC-3'; OCT4 forward, 5'-AGCTTGGGCTCG AGAAGGAT-3' and reverse, 5'-AGAGTGGTGACGGAGA CAGG-3'; NANOG forward, 5'-ACAACTGGCCGAAGA ATAGCA-3' and reverse, 5'-GGTTCCCAGTCGGGTTCA C-3'; LGR5 forward, 5'-CTCCCAGGTCTGGTGTGTTG-3' and reverse, 5'-GAGGTCTAGGTAGGAGGTGAAG-3'; and dipeptidase 1 (DPEP1) forward, 5'-CAAGTGGCCGACCAT CTGG-3' and reverse, 5'-GGGACCCTTGGAACACCATC-3'.

RNA-seq. Total RNA from human CRC HCT116 cell line was determined by measuring the optical density (OD)260/OD280 $(>1.8)$ and OD260/OD230 (>1.6), respectively. Yield and quality were assessed using an Agilent 2100 Bioanalyzer (Agilent Technologies, Inc. USA). After the sample quality control procedures, mRNA from human cell line HCT116 was enriched using oligo(dT) beads. First, mRNA was fragmented randomly by adding fragmentation buffer (New England BioLabs, Inc.), and then cDNA was synthesized using an mRNA template and random hexamer primers, after which a custom second-strand synthesis buffer (Illumina, Inc.), dNTPs, RNase $\mathrm{H}$ and DNA polymerase I (New England BioLabs, Inc.) were added to initiate second-strand synthesis. Secondly, after a series of terminal repair reactions, ligation and sequencing adaptor ligation, the double-stranded cDNA library was completed through size selection and PCR enrichment. The 
libraries were pooled and analyzed on an Illumina sequencer using the paired-end $150 \mathrm{bp}$ RapidRun format to generate 20 million total reads per sample. Raw RNA-seq reads from the sequencing instrument were first trimmed based on the low-quality tranche and were checked. Spliced Transcripts Alignment to a Reference software STAR_2.5.2b (Illumina, Inc.) was used to map spliced short-reads (RNA-seq reads) to the reference genome (Ensembl GRCh38; http://asia.ensembl. org/Homo_sapiens/Info/Index). Based on spliced alignments, transcript reconstruction and estimations of transcript abundance were conducted using Cuffquant (http://cole-trapnell-lab.github.io/cufflinks/). Gene expression was normalized by calculating the number of RNA-seq fragments per kilobase of transcript per total million fragments mapped. Cuffdiff (version v2.2.1) was used to test the statistical significance of observed changes and identify genes that were differentially regulated at the transcriptional or post-transcriptional levels (http://cole-trapnell-lab.github.io/cufflinks/).

Western blotting. Cells were washed with ice-cold PBS. Total protein was extracted with radioimmunoprecipitation assay buffer (EMD Millipore) supplemented with protease and phosphatase inhibitors (Roche Diagnostics $\mathrm{GmbH}$ ). Protein concentrations were determined using a Bradford protein assay kit (Bio-Rad Laboratories, Inc.). A total of $30 \mu \mathrm{g}$ protein/lane was separated on a $10 \%$ gel using SDS-PAGE and transferred onto Immunobilon-P polyvinylidene difluoride membranes (Merck KGaA). The membranes were washed in PBS with $10 \%$ Tween 20 (PBST). After blocking with 5\% skimmed milk in PBST for $1 \mathrm{~h}$ at room temperature, the membranes were incubated with anti-Nanog (1:500; cat. no. SC-293121; Santa Cruz Biotechnology, Inc.), anti-DPEP1 (1:1,000; cat. no. 38797; Signalway Antibody LLC) and anti-GAPDH (1:5,000; cat. no. GTX627408; GeneTex International Corporation) antibodies overnight at $4^{\circ} \mathrm{C}$. After incubation with the corresponding horseradish peroxidase-conjugated secondary antibody (anti-rabbit, cat. no. A120-101P; anti-mouse, cat. no. A90-116P; 1:5,000; Bethyl Laboratories, Inc.) for $1 \mathrm{~h}$ at room temperature, immunoreactive proteins were detected using an enhanced chemiluminescence detection system (EMD Millipore).

Lentivirus production and transduction. The stable embryonic kidney cell 293T obtained from the Bioresource Collection and Research Center was used for lentiviral production. The short hairpin (sh)RNA plasmids targeting DPEP1 (shDPEP1 \#1 is clone TRCN0000046649 and shDPEP1 \#2 is clone TRCN0000441304) and non-targeting shRNA plasmids (pLAS.Void; scrambled sequence, 5'-AGTTCAGTTACGATATCATGT-3') were obtained from the National RNAi Core Facility (Taipei, Taiwan) and prepared for lentiviral transduction. TurboFect ${ }^{\mathrm{TM}}$ Transfection Reagent (Thermo Fisher Scientific, Inc) was used for transfection in accordance with the manufacturers' protocol. Initially, lentiviruses were produced and collected after $24 \mathrm{~h}$ transduction with $293 \mathrm{~T}$ cells. After $24 \mathrm{~h}$ of infection, cells (G8D-shDPEP1) were treated with puromycin $(2 \mu \mathrm{g} / \mathrm{ml})$ to select for a pool of drug-resistant clones.

Xenograft tumorigenicity assay. All animal experimental procedures and methods were performed in accordance with the relevant guidelines and regulations of the $3 \mathrm{Rs}$ and animal welfare. All animal protocols were approved by the Academia Sinica Institutional Animal Care and Utilization Committee (Protocol ID, 18-08-1222). Mice were housed in washed polysulfone micro-isolator cages in a specific-pathogen-free facility, with controlled light (12 h light/dark), constant temperature $\left(22^{\circ} \mathrm{C}\right)$ and humidity $(55 \pm 5 \%)$, ventilation rate of 15 times/h and were provided standard mouse chow (Autoclavable Rodent Diet 5010; LabDiet) and water ad libitum. Xenograft tumorigenicity was determined as previously described (20). Briefly, HCT116 cells at different tumorsphere generations (G0 or G8D) or with different treatments [G8D with scramble shRNA (non-targeting negative control shRNA) or shDPEP1 viral infection] were harvested, washed with PBS and resuspended in DMEM/F12 medium. Cells $\left(1 \times 10^{2}\right)$ were injected subcutaneously into the right and left flank regions of 6-week-old male NOD/SCID/ $\lambda$ (NSG) mice (Genomic Research Center, Taipei, Taiwan). A total of 19 mice were used $(\sim 25 \mathrm{~g})$. The mice were injected on both flank regions and divided into two groups. The first group of mice ( $n=9)$ was injected with G0 on the left and G8D on the right. The second group of mice $(n=10)$ was injected with shDPEP1 on the left and scramble shRNA on the right. The maxima observed tumor size was $\sim 0.6 \mathrm{~cm}^{3} . \mathrm{CO}_{2}$ was used as the euthanasia agent and delivered to the cage in a controllable fashion at a flow rate of $30 \%$ volume displacement per min. All mice were euthanized 28 days after injection, the tumors were surgically excised and weighed, and their volume was measured. Tumor volumes were calculated according to the formula $[(\pi / 6) \mathrm{x}$ width $2 \mathrm{x}$ length].

Statistical analysis. All statistical analyses were performed using GraphPad Prism (v.6.0; GraphPad Software, Inc.). Unpaired t-test was used to determine differences between 2 groups. One-way ANOVA followed by Tukey's post hoc test was used to compare differences among $\geq 3$ groups, whereas ANOVA followed by Dunnett's post hoc test was used to compare $\geq 2$ experimental groups with a single control. $\mathrm{P}<0.05$ was considered to indicate a statistically significant difference.

\section{Results}

Selection of a cancer cell line using repeated sphere-forming assays. The experimental design of the present study is illustrated in Fig. 1. Using an ultra-low attachment dish, tumorspheres were generated using the sphere-forming assay. Whereas the majority of cells died during culture, a small population (CSC-like cells) was able to form spheres. These SDCSCs were isolated, and a portion was collected and subjected to experimental validation. The remaining cells were dissociated and re-plated onto a regular dish as SDACs. Some SDACs were frozen in stock vials at $-80^{\circ} \mathrm{C}$, while others were allowed to expand, and after recovery for 14 days, cells were again cultured in an ultra-low attachment dish to form spheres for the next round of selection (Fig. 1). For simplicity, 'generation' was abbreviated as 'G', 'SDCSCs' as 'S' and 'SDACs' as 'D'. For example, first-generation SDCSCs were designated as 'G1S' and first-generation SDACs as 'G1D'.

The human CRC HCT116 cell line was used as the model cell line in the present study. At the beginning of the assay, HCT116 cells were subjected to a one-time homogenizing 


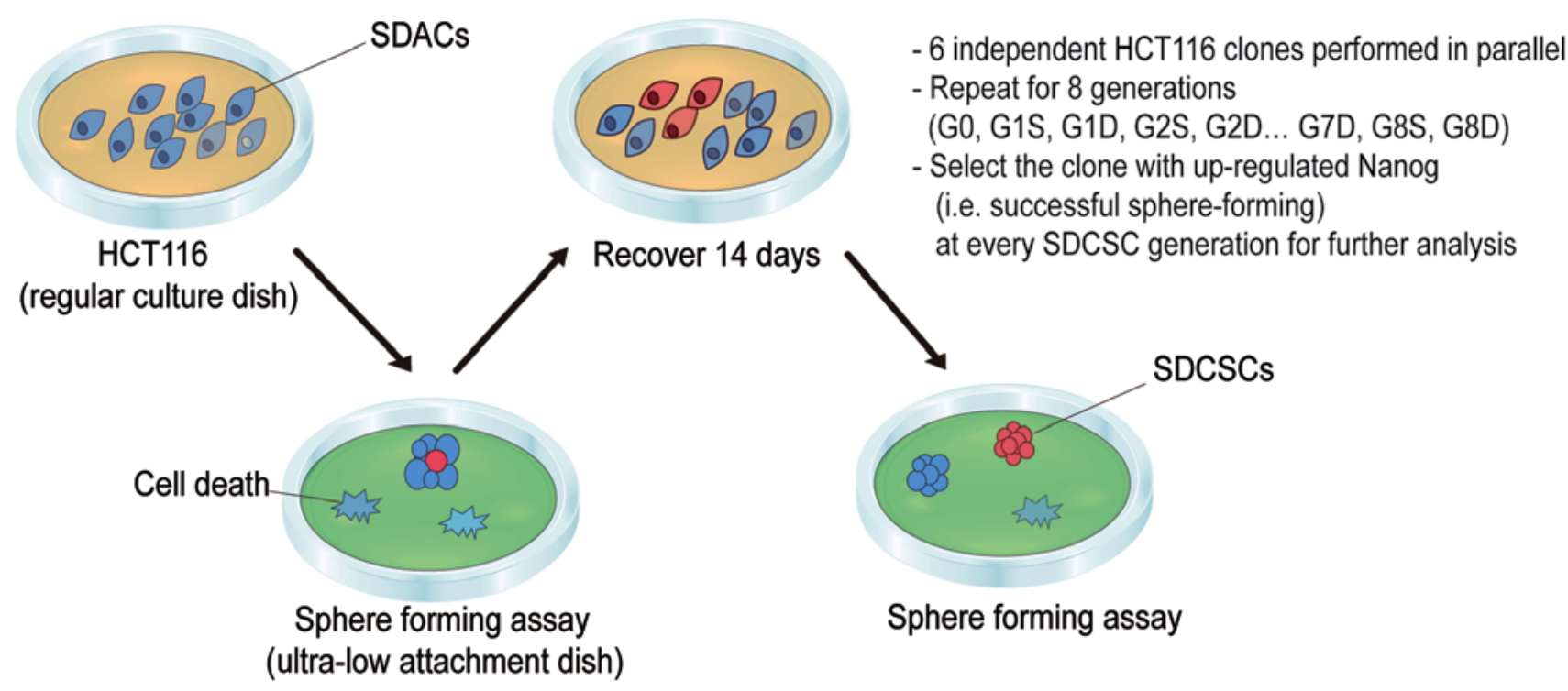

Figure 1. Schematic representation of the repeated sphere-forming assay to generate SDCSCs and SDACs. At the beginning of the repeated sphere-forming and recovery assay, the majority of cells died upon seeding onto ultra-low attachment dishes, with few cells being able to form SDCSCs. SDCSCs were dissociated to obtain single cells and subsequently re-plated onto a regular dish as SDACs for recovery. After a 14-day recovery, SDACs were seeded onto ultra-low attachment dishes again to form SDCSCs. Six independent HCT116 clones were subjected to the sphere-forming and recovery procedures in parallel for eight repeated generations. The HCT116 clone confirmed based on Nanog upregulation at every generation was selected for further analysis. Orange dish, regular culture dish; green dish, ultra-low attachment dish. SDCSCs/S, sphere-derived cancer stem cell-like cells; SDACs/D, sphere-derived adherent cells; G, generation.

sphere-forming assay and re-plated as adherent cells. These homogenized HCT116 cells were considered parental and designated 'G0'. Beginning with this parental line, repeated sphere-forming assays and recovery procedures were performed for the generation of SDCSCs and SDACs. A total of six independent HCT116 clones were subjected to the sphere-forming and recovery procedures in parallel for eight repeated stages. The eighth-generation SDCSCs and SDACs were designated as G8S and G8D, respectively.

Canonical stemness markers are transiently expressed in SDCSCs. To validate that SDCSCs generated from the aforementioned repeated sphere-forming assay were CSC-like tumorspheres at every generation, the expression levels of the canonical stemness marker Nanog (21) were analyzed via RT-qPCR. SDACs and SDCSCs from G0 (parental) to G8 were collected. Among the six independent HCT116 clones, one clone (clone 1) exhibited a marked upregulation of Nanog expression in SDCSCs at every generation compared with Nanog expression at G0 (Fig. 2A). In addition, another HCT116 clone (clone 2) exhibited a similar upregulation of Nanog expression in SDCSCs at six out of eight generations (Fig. S1; except for the first and second generations, where the Nanog levels were comparable in SDCSCs and SDACs). The variation in Nanog expression may result from differences in sphere-forming efficiency. Since Nanog upregulation was observed at every generation in clone 1 , this was selected for further analysis. The upregulation of Nanog in SDCSCs appeared to only be transient, as the Nanog expression level in SDACs at every generation was close to that in G0 SDACs (Fig. 2A). Subsequently, a time-course experiment was performed. The G1S spheres were collected and re-plated onto a regular dish as adherent cells that were harvested at days 1, 3, 7 and 14. Nanog expression was markedly increased in SDACs at day 1 compared with SDCSCs, but gradually decreased from days 3 to 14 in SDACs, at both the mRNA (Fig. 2B; $\mathrm{P}<0.0001, \mathrm{P}<0.05, \mathrm{P}<0.05$ and $\mathrm{P}<0.01$ for day 1,3, 7 and 14, respectively) and protein levels (Fig. 2C). This transient pattern suggested that the upregulation of this marker may be a consequence of the sphere-forming assay and may therefore depend on the sphere state, rather than being a stable phenotype of the cells. In addition, we also observed similar transient upregulation of stemness genes in SDCSCs using a stem cell marker of the intestinal epithelium, LGR5 (22), and another pluripotent marker, OCT4 (21) (Fig. 2D). This result indicated that such transient upregulation was not limited to a single stemness gene.

Sphere-forming capacity increases significantly after eight rounds of selection. In order to analyze whether repetitive rounds of selection would result in a phenotypically more malignant cell line, the sphere-forming capacities of first-, fourth- and eighth-generation cells were compared in vitro. While the morphologies of SDACs were similar among G1D, G4D and G8D cells (Fig. 3A), the size and number of spheres that formed increased gradually from G1S to G4S to G8S $(\mathrm{P}<0.05$; Fig. $3 \mathrm{~B}$ and $\mathrm{C})$. The present in vitro validation confirmed that the repeated sphere-forming and recovery procedures were able to generate functionally more malignant cells.

RNA sequencing identifies DPEPI as a highly expressed gene in the selected clone. The increased sphere-forming capacity at G8 suggested that the present repeated sphere-forming assay was able to select for a more malignant subpopulation (Fig. 3). Therefore, the present study aimed to identify any stably 
A

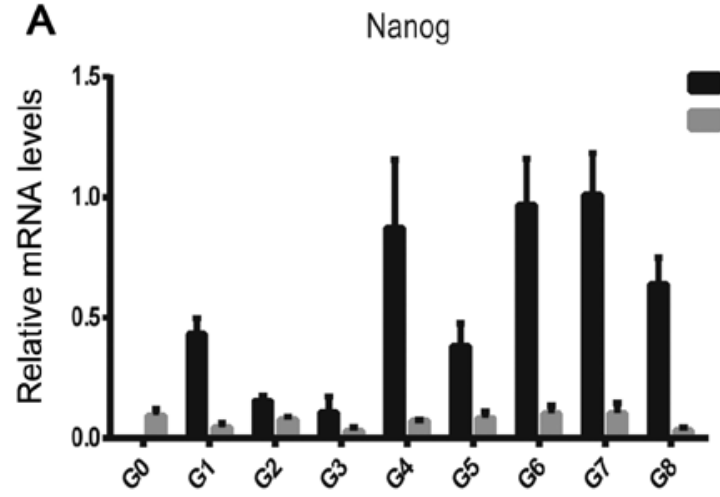

C

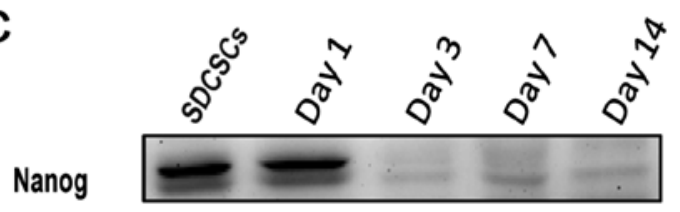

GAPDH
B

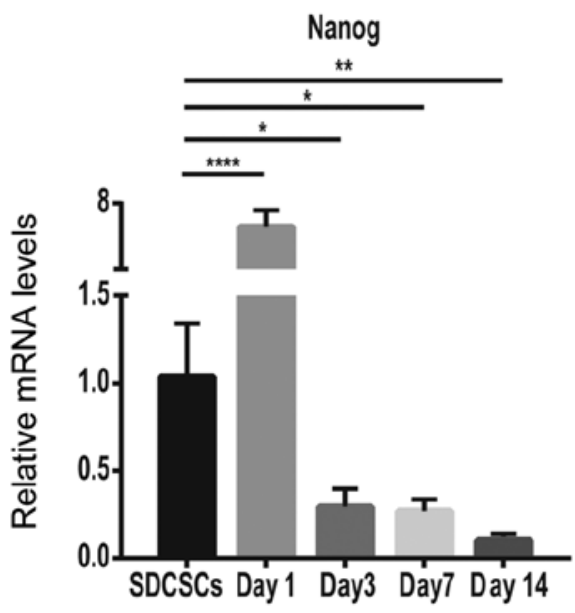

SDCSCS

SDACS
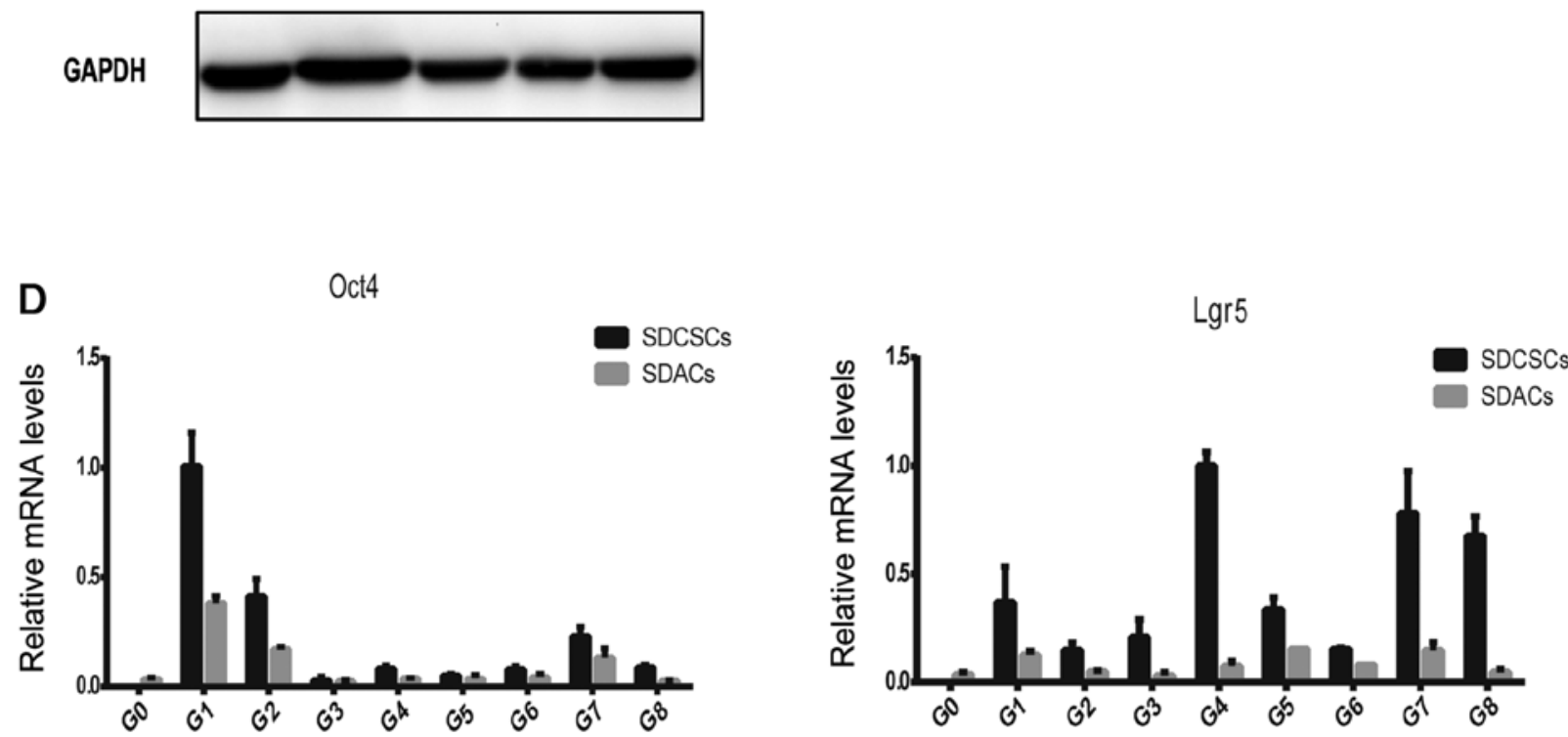

Figure 2. Validation of the repeated sphere-forming and recovery assay using stemness markers. (A) Expression levels of Nanog mRNA in SDCSCs and SDACs from G0 (parental) to G8 were analyzed via RT-qPCR. (B) RNA was collected from SDCSCs at days 1, 3, 7 and 14 after re-plating as SDACs. The expression levels of Nanog mRNA were analyzed via RT-qPCR. Significant differences were determined by ANOVA followed by Dunnett's post hoc test. ${ }^{*} \mathrm{P}<0.05 ;{ }^{* *} \mathrm{P}<0.01 ;{ }^{* * * * *} \mathrm{P}<0.0001$. (C) Proteins were collected from SDCSCs at days 1, 3, 7 and 14 after re-plating as SDACs. Nanog protein expression was detected by western blotting. GAPDH was used as the internal control. (D) Expression levels of Oct4 and Lgr5 mRNA in SDCSCs and SDACs from G0 (parental) to G8, as analyzed by RT-qPCR. Data are presented as the mean \pm SD $(n=3)$. GAPDH was used as the reference gene. SDCSCs, sphere-derived cancer stem cell-like cells; SDACs, sphere-derived adherent cells; G, generation; RT-qPCR, reverse transcription-quantitative PCR; Oct4, octamer-binding transcription factor 4; Lgr5, leucine-rich repeat-containing G-protein coupled receptor 5.

upregulated genes in the selected clone that may be responsible for this phenotype. Compared with G0 cells, RNA sequencing was performed to identify genes differentially expressed in G8D, including 202 significantly upregulated genes (red dots) and 289 downregulated genes (blue dots; Fig. 4A). Candidate genes were selected based on the differential expression in both sphere and adherent states (G8S > G1S > G0 and G8D > G0), and the top 10 upregulated or downregulated protein-coding genes are listed in Fig. 4B. DPEP1, the top-ranked gene that encodes the DPEP1 enzyme, which has been implicated in the progression of colon cancer malignancy (23-25), was selected for further analysis. The upregulation of DPEP1 mRNA in G8D cells was confirmed via RT-qPCR ( $\mathrm{P}<0.05$; Fig. 4C). In addition, DPEP1 was similarly upregulated in clone $2(\mathrm{P}<0.01$; Fig. S2). There were two different lentiviral shRNAs that were used to stably knockdown DPEP1 in G8D cells in the present study. Knockdown efficiency was confirmed at both the mRNA (P<0.01 for shDPEP1 \#1 and \#2; Fig. 4D) and protein levels (Fig. 4E). DPEP1 knockdown led to a significant decrease in the sphere-forming capacity of G8D cells (Fig. 4F; P $<0.0001$ and $\mathrm{P}<0.05$ for shDPEP1 \#1 and \#2, respectively; Fig. 4G; $\mathrm{P}<0.0001$ for shDPEP1 \#1 and \#2), suggesting that the high 

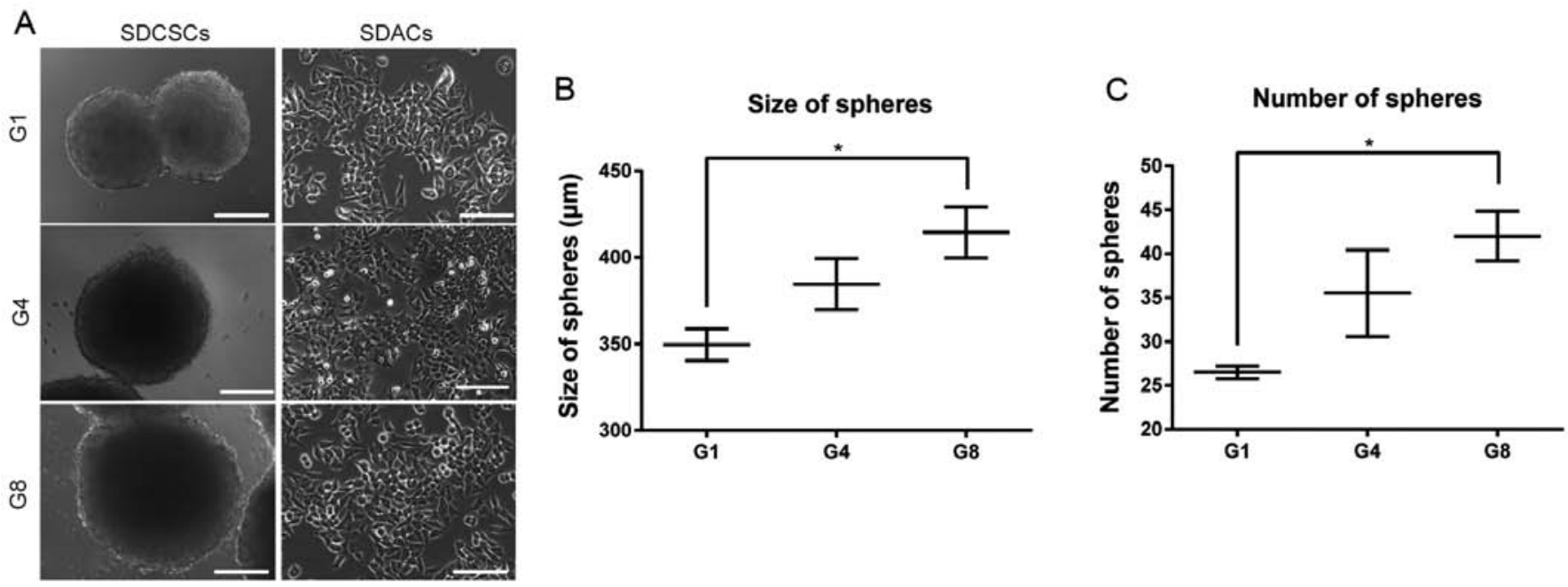

Figure 3. Increased sphere-forming capacity at later generations. (A) Representative phase-contrast images of SDCSCs and SDACs at G1, G4 and G8 stages. SDCSCs isolated from the sphere-forming assay were allowed to recover for 14 days to obtain SDACs. Scale bar, $100 \mu \mathrm{m}$ (SDCSCs) and $50 \mu \mathrm{m}$ (SDACs). Quantification of (B) size (diameter) and (C) number of spheres at G1, G4 and G8. For the number of spheres, the average numbers from triplicate wells are shown. For the size of spheres, $>80$ cells were analyzed in every condition. HCT116 clones 1 and 2 served as two independent experiments. Data are presented as the mean \pm SD. Significant differences were determined by ANOVA followed by Tukey's post hoc test. "P $<0.05$. SDCSCs, sphere-derived cancer stem cell-like cells; SDACs, sphere-derived adherent cells; G, generation.

expression level of DPEP1 may promote colorectal cancer malignancy.

Eighth-generationSDACsexhibitsenhancedDPEPl-dependent tumorigenicity in vivo. To investigate whether the selected clone possessed enhanced DPEP1-dependent tumorigenicity in vivo, tumor growth was examined in a xenograft model using severely immunodeficient NSG mice (26). As presented in Fig. 5A, tumor size (e.g., tumor weight and volume) was markedly increased using G8D cells when compared with using G0 cells, indicating a stronger tumorigenicity after eight rounds of selection. Additionally, DPEP1-knockdown in G8D cells greatly decreased tumorigenicity, which was similar to that using G0 cells (Fig. 5A-C; $\mathrm{P}<0.05, \mathrm{P}<0.001$ and $\mathrm{P}<0.01$ for G0 vs. G8D, G8D vs. shDPEP1 and shDPEP1 vs. scramble shRNA, respectively; Fig. $5 \mathrm{C} ; \mathrm{P}<0.05, \mathrm{P}<0.01$ and $\mathrm{P}<0.05$ for G0 vs. G8D, G8D vs. shDPEP1 and shDPEP1 vs. scramble shRNA, respectively). Overall, these data confirmed that the repeated sphere-forming strategy used in the present study may successfully select a highly tumorigenic clone. DPEP1 was stably upregulated in G8D cells and may be responsible for the elevated tumorigenicity.

\section{Discussion}

Stem cells are defined by their ability to self-renew and differentiate into a variety of cell types (27). The concept has expanded from embryonic stem cells to adult stem cells to CSCs (28). The sphere-forming assay is traditionally used to identify cells that possess stem cell characteristics (8), as well as to characterize CSCs (9). Previously, when cancer cells were cultured as tumorspheres (CSC-like cells), later generations were reported to be more tumorigenic than parental cells (29). However, since cells in tumorspheres are temporarily cultured in a condition that is different from their long-term adherent culture, it is likely that the phenotypes measured merely reflect an acute state. In other words, the expression levels of stemness markers (e.g. SRY-Box Transcription Factor 2, OCT4 and Nanog) and properties (e.g. tumor-initiating capability, multi-drug resistance) may only be transient (15). The present study indicated that the expression levels of the three canonical stem cell markers, Nanog, Oct4 and Lgr5, were upregulated in tumorspheres (SDCSCs), but their expression decreased to levels close to parental ones when the spheres were re-plated as SDACs. Based on the present finding, an alternative 'intermittent' strategy was adopted. Specifically, after 14 days of sphere formation, cells were allowed to rest in the adherent state for 14 days before the next sphere-forming assay. Using this experimental strategy, cell lines were generated and stable phenotypes were identified. Another benefit of the present strategy was that cells could be expanded and stored in the resting SDAC state and could be recovered at every generation, in contrast to continuous propagation as with tumorspheres (29), which only lasted for a limited duration (data not shown). Theoretically, the assay may be performed for infinite generations using the intermittent strategy.

Using the intermittent strategy, a clone was isolated after eight rounds of sphere formation that was more tumorigenic in its adherent state than the parental line. Since the number of selection rounds was relatively small, it is probable that the selected clone $(\mathrm{G} 8 \mathrm{D})$ represented a pre-existing subpopulation within the heterogeneous parental line. However, given the genomic instability of cancer cells, it is likely that increasing the number of selection rounds may allow for the accumulation of new mutations during the course of the experiment. Therefore, the present system has the potential to be used in 'experimental cancer evolution' studies, as being proposed in a previous perspective article (30). In this case, the hypoxic microenvironment within the tumorsphere may serve as the selection pressure that drives evolution towards a potentially more devastating 
A

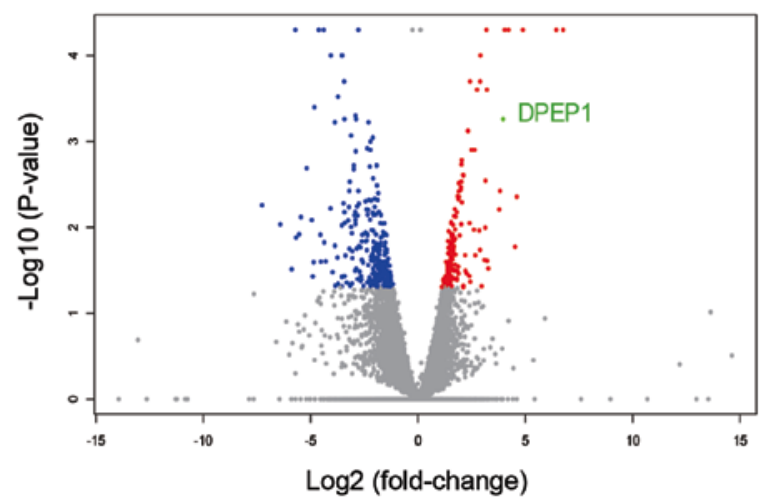

B

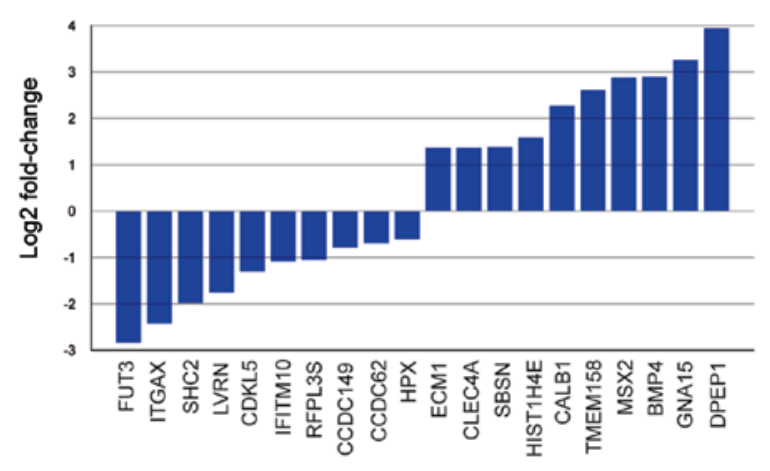

D

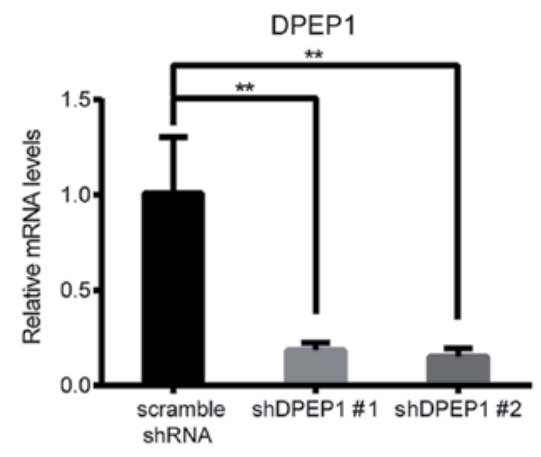

$\mathbf{F}$

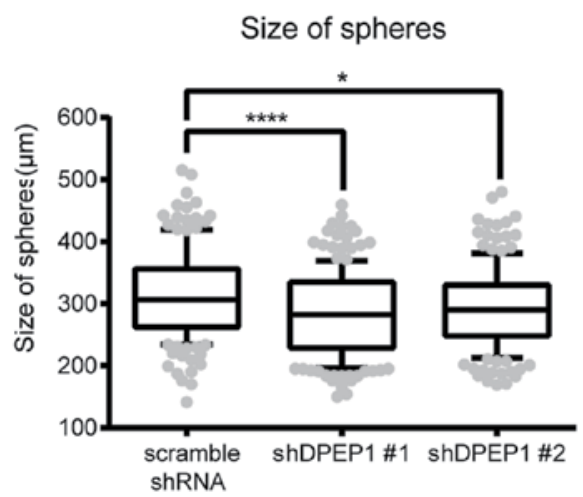

C

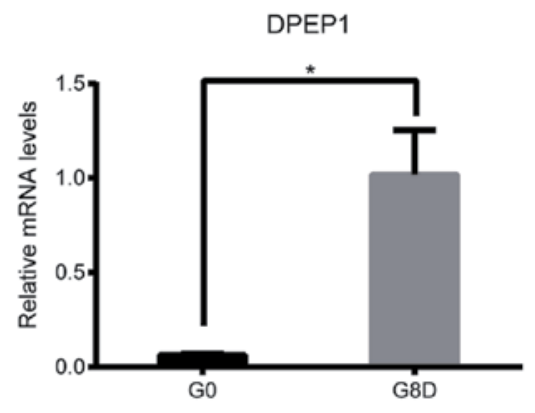

E

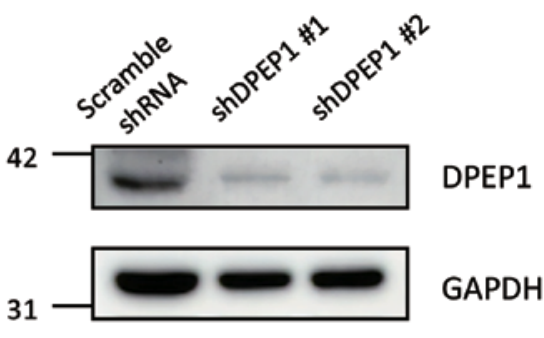

G Number of spheres

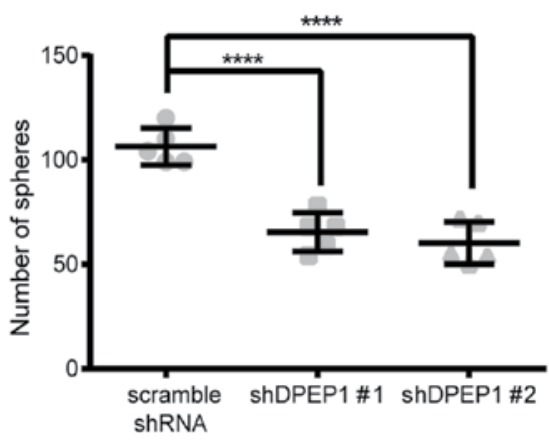

Figure 4. DPEP1 promotes sphere-formation in G8D. (A) Volcano plot comparing G8D and G0 stages based on RNA sequencing data. DPEP1 (marked in green) ranked as one of the most significantly upregulated genes among all probes. Red dots represent upregulated genes and blue dots represent downregulated genes. (B) DPEP1 was the top upregulated protein-coding gene. The top 10 upregulated or downregulated protein-coding genes were selected based on the following criteria: G8S > G1S > G0 and G8D > G0. In other words, differential expression in both sphere and adherent states were applied for this selection. (C) Differential expression of DPEP1 mRNA between G0 (parental) to G8 cells was confirmed via RT-qPCR. Data are presented as the mean $\pm \mathrm{SD}(\mathrm{n}=3)$. GAPDH was used as the reference gene. Significant differences were determined using unpaired t-test. Knockdown of DPEP1. (D) mRNA and (E) protein expression was confirmed by RT-qPCR and western blotting, respectively. GAPDH was used as the internal control. mRNA data are presented as the mean $\pm \mathrm{SD}(\mathrm{n}=3)$. Significant differences were determined using ANOVA followed by Dunnett's post hoc test. Quantification of $(\mathrm{F})$ size and $(\mathrm{G})$ number of spheres following treatment with scramble shRNA or shDPEP1 \#1/2 (n>120 cells analyzed in both G1 and G8 stages). Fig. 4F is analyzed and shown in box and whisker plots, and the whiskers were drawn down to the 10th percentile and up to the 90th. Fig. $4 \mathrm{G}$ is presented as the mean $\pm \mathrm{SD}$. Significant differences were determined using ANOVA followed by Dunnett's post hoc test. ${ }^{*} \mathrm{P}<0.05 ;{ }^{* *} \mathrm{P}<0.01 ;{ }^{* * * * *} \mathrm{P}<0.0001$. DPEP1, dipeptidase 1; G, generation; D, sphere-derived adherent cells; S, sphere-derived cancer stem cell-like cells; RT-qPCR, reverse transcription-quantitative PCR; sh, short hairpin. 
A

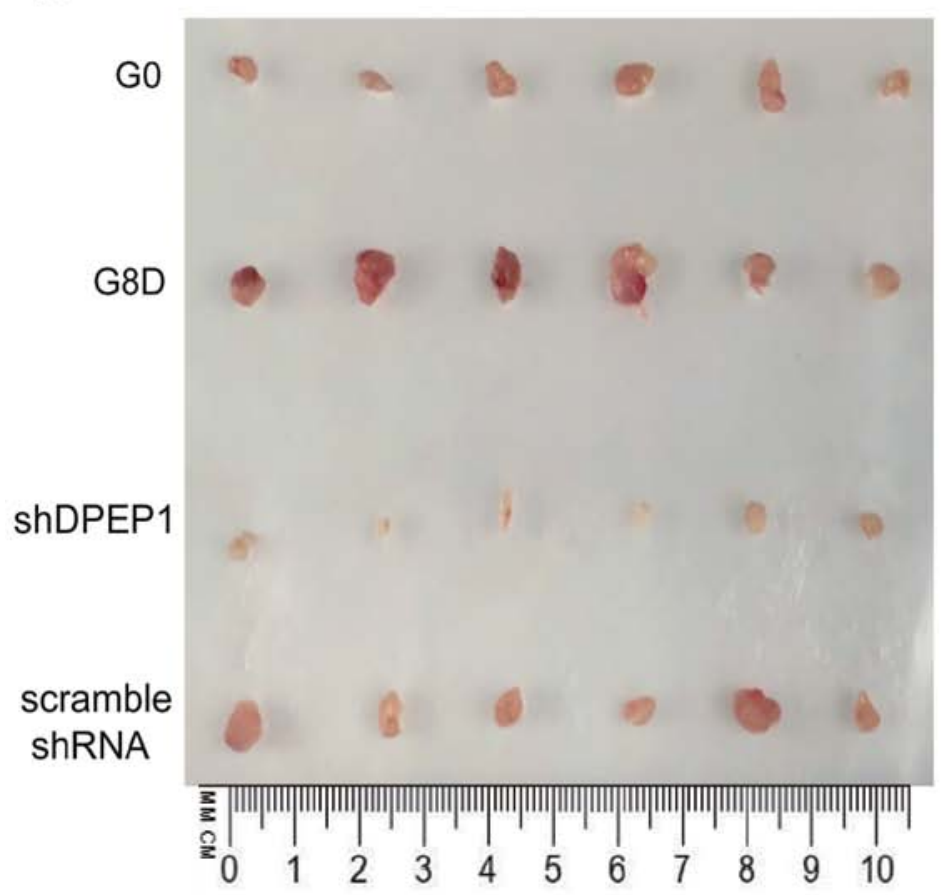

B
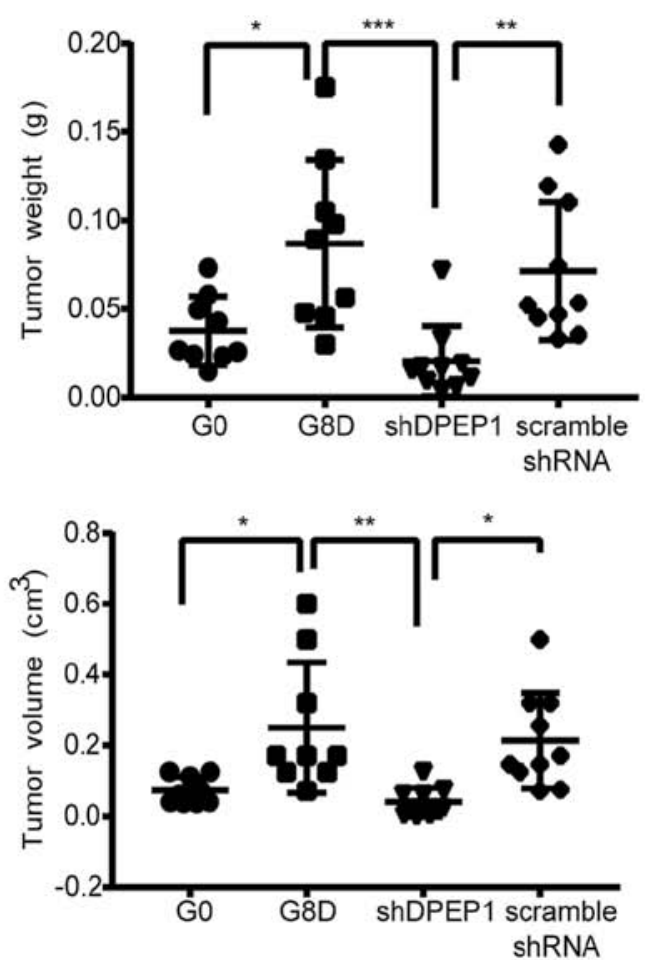

Figure 5. G8D exhibit higher DPEP1-dependent tumorigenicity in vivo. (A) Representative images of excised tumors. (B) Quantification of excised tumor weights, and (C) tumor volumes calculated according to the formula [( $\pi / 6) \times$ width $_{2} \times$ length] for the indicated treatments as follows: G0 ( $=9$ ), G8D ( $=9$ ), G8-shDPEP1 $(n=10)$ and G8-scrambled shRNA $(n=10)$. Significant differences were determined using ANOVA followed by Tukey's post hoc test. * $<0.05$; ${ }^{* *} \mathrm{P}<0.01 ;{ }^{* * *} \mathrm{P}<0.001$. G8D, eighth-generation sphere-derived adherent cells; DPEP1, dipeptidase 1; sh, short hairpin.

phenotype (31). Given the previous success achieved with the study of the experimental evolution of multicellularity (stemness) from unicellular Saccharomyces cerevisiae (32) and Chlamydomonas (33), it seems reasonable that it may be possible to experimentally induce the evolution of stable stemness in cancer and produce a stable CSC-like line, which would be a good experimental model for drug screening for anti-CSC agents.

To demonstrate that the present procedure may be valuable to identify cancer-associated genes, a transcriptomic analysis was performed, identifying DPEP1 as the top differentially expressed gene. DPEP1 was first identified as a tumor suppressor in the kidney (34) and it has been negatively associated with the development of breast (35) and pancreatic carcinoma (36). However, in CRC, DPEP1 is considered to be a positive regulator, as its expression levels are higher in tumors compared with those in paired adjacent colorectal tissues (23), and in tumor tissues compared with those in normal mucosa $(24,25)$. Additionally, high DPEP1 levels are associated with less survival time in patients with CRC (23). In the present study, DPEP1 was responsible for the elevated sphere-forming capacity in vitro and tumorigenicity in vivo during evolution of the HCT116 clone. The current results support the view that high DPEP1 levels are associated with CRC malignancy and further strengthen the concept of using DPEP1 expression as a CRC prognostic marker. Mechanistically, it has been reported that DPEP1 promotes cell proliferation in vitro (23). Since DPEP1 is a matrix metalloproteinase, it may also promote tumor growth in vivo by degrading matrix barriers to enhance cell migration and angiogenesis (37). Further investigations are required to clarify the contribution of DPEP1 to in vivo tumor growth.

\section{Acknowledgements}

The authors would like to thank Technology Commons (College of Life Science, National Taiwan University) for use of the Leica TCS SP5 confocal microscope. The authors would also like to thank the Pathology Core Laboratory (Academia Sinica) for assisting with the animal experiments.

\section{Funding}

The present study was funded by the Ministry of Science and Technology (grant nos. 102-2311-B-002-041-MY3, 108-3114-Y-001-002, 108-2811-B-001-581, 109-0210-01-18-02, 108-2314-B-001-006-MY3) and Academia Sinica (grant nos. AS-SUMMIT-108, AS-SUMMIT-109 and AS-KPQ-109-BioMed).

\section{Availability of data and materials}

The datasets used and/or analyzed during the present study are available from the corresponding author on reasonable request.

\section{Authors' contributions}

PL performed the animal experiments and analyzed the RNA-seq data. TC performed the repeated sphere-forming 
experiments. CF characterized DPEP1. HL performed animal experiments. YC performed the lentiviral knockdown experiments. $\mathrm{CN}$ and $\mathrm{MH}$ assisted in the animal experiments. PL, $\mathrm{JL}$ and $\mathrm{HH}$ directed the project, analyzed and interpreted the data, and wrote the manuscript. All authors read and approved the final manuscript.

\section{Ethics approval and consent to participate}

All animal experimental procedures and methods were performed in accordance with the relevant guidelines and regulations. All animal protocols were approved by Academia Sinica Institutional Animal Care and Utilization Committee (Protocol ID, 18-08-1222).

\section{Patient consent for publication}

Not applicable.

\section{Competing interests}

The authors declare that they have no competing interests.

\section{References}

1. Siegel RL, Miller KD and Jemal A: Cancer statistics, 2020. CA Cancer J Clin 70: 7-30, 2020.

2. Bhandari A, Woodhouse M and Gupta S: Colorectal cancer is a leading cause of cancer incidence and mortality among adults younger than 50 years in the USA: A SEER-based analysis with comparison to other young-onset cancers. J Investig Med 65: 311-315, 2017.

3. Harrison $\mathrm{S}$ and Benziger $\mathrm{H}$ : The molecular biology of colorectal carcinoma and its implications: A review. Surgeon 9: 200-210, 2011

4. Siegel R, Ward E, Brawley O and Jemal A: Cancer statistics, 2011: The impact of eliminating socioeconomic and racial disparities on premature cancer deaths. CA Cancer J Clin 61: 212-236, 2011

5. Vaiopoulos AG, Kostakis ID, Koutsilieris M and Papavassiliou AG: Colorectal cancer stem cells. Stem Cells 30: 363-371, 2012.

6. Dean M, Fojo T and Bates S: Tumour stem cells and drug resistance. Nat Rev Cancer 5: 275-284, 2005.

7. Altman J: Autoradiographic and histological studies of postnatal neurogenesis. IV. Cell proliferation and migration in the anterior forebrain, with special reference to persisting neurogenesis in the olfactory bulb. J Comp Neurol 137: 433-457, 1969.

8. Pastrana E, Silva-Vargas V and Doetsch F: Eyes wide open: A critical review of sphere-formation as an assay for stem cells. Cell Stem Cell 8: 486-498, 2011.

9. Bahmad HF, Cheaito K, Chalhoub RM, Hadadeh O, Monzer A, Ballout F, El-Hajj A, Mukherji D, Liu YN, Daoud G and Abou-Kheir W: Sphere-formation assay: Three-dimensional in vitro culturing of prostate cancer stem/progenitor sphere-forming cells. Front Oncol 8: 347, 2018.

10. Singh SK, Clarke ID, Terasaki M, Bonn VE, Hawkins C, Squire J and Dirks PB: Identification of a cancer stem cell in human brain tumors. Cancer Res 63: 5821-5828, 2003.

11. Ponti D, Costa A, Zaffaroni N, Pratesi G, Petrangolini G, Coradini D, Pilotti S, Pierotti MA and Daidone MG: Isolation and in vitro propagation of tumorigenic breast cancer cells with stem/progenitor cell properties. Cancer Res 65: 5506-5511, 2005

12. Ricci-Vitiani L, Lombardi DG, Pilozzi E, Biffoni M, Todaro M, Peschle C and De Maria R: Identification and expansion of human colon-cancer-initiating cells. Nature 445: 111-115, 2007.

13. Todaro M, Alea MP, Di Stefano AB, Cammareri P, Vermeulen L, Iovino F, Tripodo C, Russo A, Gulotta G, Medema JP and Stassi G: Colon cancer stem cells dictate tumor growth and resist cell death by production of interleukin-4. Cell Stem Cell 1: 389-402, 2007.

14. Amsterdam A, Raanan C, Schreiber L, Freyhan O, Fabrikant Y, Melzer E and Givol D: Differential localization of LGR5 and Nanog in clusters of colon cancer stem cells. Acta Histochem 115: $320-329,2013$
15. Yakisich JS, Azad N, Kaushik V and Iyer AKV: Cancer cell plasticity: Rapid reversal of chemosensitivity and expression of stemness markers in lung and breast cancer tumorspheres. J Cell Physiol 232: 2280-2286, 2017.

16. Chu YW, Yang PC, Yang SC, Shyu YC, Hendrix MJ, Wu R and Wu CW: Selection of invasive and metastatic subpopulations from a human lung adenocarcinoma cell line. Am J Respir Cell Mol Biol 17: 353-360, 1997.

17. Yang CF, Tsai WY, Chen WA, Liang KW, Pan CJ, Lai PL, Yang PC and Huang HC: Kinesin-5 Contributes to Spindle-length scaling in the evolution of cancer toward metastasis. Sci Rep 6: 35767, 2016.

18. Chen JJ, Peck K, Hong TM, Yang SC, Sher YP, Shih JY, Wu R, Cheng JL, Roffler SR, Wu CW and Yang PC: Global analysis of gene expression in invasion by a lung cancer model. Cancer Res 61: 5223-5230, 2001.

19. Livak KJ and Schmittgen TD: Analysis of relative gene expression data using real-time quantitative PCR and the 2(-Delta Delta $\mathrm{C}(\mathrm{T})$ ) method. Methods 25: 402-408, 2001.

20. Morton CL and Houghton PJ: Establishment of human tumor xenografts in immunodeficient mice. Nat Protoc 2: 247-250, 2007.

21. Liu A, Yu X and Liu S: Pluripotency transcription factors and cancer stem cells: Small genes make a big difference. Chin J Cancer 32: 483-487, 2013.

22. de Sousa e Melo F, Kurtova AV, Harnoss JM, Kljavin N, Hoeck JD, Hung J, Anderson JE, Storm EE, Modrusan Z, Koeppen H, et al: A distinct role for Lgr5(+) stem cells in primary and metastatic colon cancer. Nature 543: 676-680, 2017.

23. Hao JJ, Zhi X, Wang Y, Zhang Z, Hao Z, Ye R, Tang Z, Qian F, Wang Q and Zhu J: Comprehensive proteomic characterization of the human colorectal carcinoma reveals signature proteins and perturbed pathways. Sci Rep 7: 42436, 2017.

24. Eisenach PA, Soeth E, Röder C, Klöppel G, Tepel J, Kalthoff H and Sipos B: Dipeptidase 1 (DPEP1) is a marker for the transition from low-grade to high-grade intraepithelial neoplasia and an adverse prognostic factor in colorectal cancer. Br J Cancer 109: 694-703, 2013.

25. Park SY, Lee SJ, Cho HJ, Kim TW, Kim JT, Kim JW, Lee CH, Kim BY, Yeom YI, Lim JS, et al: Dehydropeptidase 1 promotes metastasis through regulation of E-cadherin expression in colon cancer. Oncotarget 7: 9501-9512, 2016.

26. Shultz LD, Lyons BL, Burzenski LM, Gott B, Chen X, Chaleff S, Kotb M, Gillies SD, King M, Mangada J, et al: Human lymphoid and myeloid cell development in NOD/LtSz-scid IL2R gamma null mice engrafted with mobilized human hemopoietic stem cells. J Immunol 174: 6477-6489, 2005.

27. Kolios G and Moodley Y: Introduction to stem cells and regenerative medicine. Respiration 85: 3-10, 2013.

28. Kim WT and Ryu CJ: Cancer stem cell surface markers on normal stem cells. BMB Rep 50: 285-298, 2017.

29. Cao L, Zhou Y, Zhai B, Liao J, Xu W, Zhang R, Li J, Zhang Y, Chen L, Qian H, et al: Sphere-forming cell subpopulations with cancer stem cell properties in human hepatoma cell lines. BMC Gastroenterol 11: 71, 2011.

30. Taylor TB, Johnson LJ, Jackson RW, Brockhurst MA and Dash PR: First steps in experimental cancer evolution. Evol Appl 6: 535-548, 2013.

31. Yun Z and Lin Q: Hypoxia and regulation of cancer cell stemness Adv Exp Med Biol 772: 41-53, 2014.

32. Ratcliff WC, Denison RF, Borrello $M$ and Travisano M: Experimental evolution of multicellularity. Proc Natl Acad Sci USA 109: 1595-1600, 2012.

33. Ratcliff WC, Herron MD, Howell K, Pentz JT, Rosenzweig F and Travisano M: Experimental evolution of an alternating Uniand multicellular life cycle in Chlamydomonas reinhardtii. Nat Commun 4: 2742, 2013.

34. Austruy E, Cohen-Salmon M, Antignac C, Béroud C, Henry I, Nguyen VC, Brugières L, Junien C and Jeanpierre C: Isolation of kidney complementary DNAs down-expressed in Wilms' tumor by a subtractive hybridization approach. Cancer Res 53: 2888-2894, 1993.

35. Green AR, Krivinskas S, Young P, Rakha EA, Paish EC, Powe DG and Ellis IO: Loss of expression of chromosome 16q genes DPEP1 and CTCF in lobular carcinoma in situ of the breast. Breast Cancer Res Treat 113: 59-66, 2009.

36. Zhang G, Schetter A, He P, Funamizu N, Gaedcke J, Ghadimi BM, Ried T,Hassan R, Yfantis HG, Lee DH, et al: DPEP1 inhibits tumor cell invasiveness, enhances chemosensitivity and predicts clinical outcome in pancreatic ductal adenocarcinoma. PLoS One 7: e31507, 2012.

37. Deryugina EI and Quigley JP: Matrix metalloproteinases and tumor metastasis. Cancer Metastasis Rev 25: 9-34, 2006.

This work is licensed under a Creative Commons Attribution-NonCommercial-NoDerivatives 4.0 International (CC BY-NC-ND 4.0) License. 